\title{
Geocoding Large Population-level Administrative Datasets at Highly Resolved Spatial Scales
}

\author{
Sharon E. Edwards, ${ }^{*}$ Benjamin Strauss ${ }^{\dagger}$ and Marie Lynn Miranda*, \\ ${ }^{*}$ Children's Environmental Health Initiative, University of Michigan \\ ${ }^{+}$Nicholas School of the Environment, Duke University \\ ${ }^{\ddagger}$ Department of Pediatrics, University of Michigan
}

\begin{abstract}
Using geographic information systems to link administrative databases with demographic, social, and environmental data allows researchers to use spatial approaches to explore relationships between exposures and health. Traditionally, spatial analysis in public health has focused on the county, ZIP code, or tract level because of limitations to geocoding at highly resolved scales. Using 2005 birth and death data from North Carolina, we examine our ability to geocode population-level datasets at three spatial resolutions - zip code, street, and parcel. We achieve high geocoding rates at all three resolutions, with statewide street geocoding rates of $88.0 \%$ for births and $93.2 \%$ for deaths. We observe differences in geocoding rates across demographics and health outcomes, with lower geocoding rates in disadvantaged populations and the most dramatic differences occurring across the urban-rural spectrum. Our results suggest that highly resolved spatial data architectures for population-level datasets are viable through geocoding individual street addresses. We recommend routinely geocoding administrative datasets to the highest spatial resolution feasible, allowing public health researchers to choose the spatial resolution used in analysis based on an understanding of the spatial dimensions of the health outcomes and exposures being investigated. Such research, however, must acknowledge how disparate geocoding success across subpopulations may affect findings.
\end{abstract}

\section{Introduction}

Geographic Information Systems (GIS) and spatial analysis are of growing importance in public health research, outreach, and policy. The increasing availability and evolving methodologies of GIS technology and spatial statistics enable researchers to explore the connections between public health endpoints and relevant demographic, social, and environmental conditions by integrating across previously disparate datasets (Krieger et al. 2005; Mindell and Barrowcliffe 2005; Bergquist and Rinaldi 2010; Robinson et al. 2010; Comer et al. 2011; Eisen and Eisen 2011; Miranda and Edwards 2011; Goldberg and Jacquez 2012).

Administrative datasets, such as birth certificates, immunizations, student enrollments, notifiable diseases, and death records, are an important resource for public health researchers, as these data often cover large populations and extended periods of time. Using GIS to display these data can reveal spatial patterns which may help generate hypotheses for future research,

\footnotetext{
Address for correspondence: Marie Lynn Miranda, Children's Environmental Health Initiative, School of Natural Resources and Environment, University of Michigan, 2046 Dana Building, 440 Church Street, Ann Arbor, MI 48109, USA. E-mail: mlmirand@umich.edu Acknowledgements: This work was supported by grants from the US Environmental Protection Agency (RD-83329301) and from the National Center for Research Resources (UL1RR024128), a component of the National Institutes of Health and the NIH Roadmap for Medical Research. Funding agencies were not involved in the decisions regarding this article or the analysis it presents. We are grateful for the data management provided by Claire Osgood and the geocoding efforts of Nancy Schneider.
} 
provide information for targeting community outreach, or motivate policy efforts and priorities. Using GIS to link these administrative databases with relevant demographic, social, and environmental data via shared geography can allow for spatial statistical approaches to explore relationships between exposures and health endpoints. With key administrative datasets containing information covering many years, these data may be even more valuable in that they can enable spatio-temporal analysis of health outcomes as populations shift and exposures change over time.

Geocoding, the process of converting address information into latitude and longitude coordinates, is the key to leveraging the valuable information already collected in administrative datasets for use in spatial analyses. Four key measures of geocoding quality have been identified: completeness, resolution, matching algorithm criteria, and positional accuracy (Goldberg and Jacquez 2012). Much of the research on geocoding has focused on the positional accuracy of different geocoding techniques (Krieger et al. 2001; Ratcliffe 2001; Bonner et al. 2003; Cayo and Talbot 2003; Bow et al. 2004; Ward et al. 2005; Duncan et al. 2011; Bell et al. 2012; Goldberg and Cockburn 2012; Healy and Gilliland 2012; Jacquez 2012). Errors in positional accuracy can lead to incorrect assignment of areal units such as Census tract or even county, leading to misclassification errors (Goldberg and Cockburn 2012; McLafferty et al. 2012). Completeness of geocoding and matching algorithm criteria are difficult to compare across many studies due to a lack of detail describing the geocoding process in many papers (Robinson et al. 2010).

At least three important advances have occurred that can lead to improvements in geocoding of administrative datasets: (1) address information in administrative datasets has become more complete and standardized; (2) reference layers have been updated, standardized, and more completely populated (Rushton et al. 2006); and (3) geocoding processes and methodologies have improved (Zandbergen and Chakraborty 2006; Goldberg et al. 2007). Inevitably, however, there are records in any dataset that cannot be geocoded, and understanding systematic issues in geocoding completeness is important for understanding the types of research that can be undertaken with a particular dataset and the bias to which such research may be subject.

In this article, we focus on two of the key measures of geocoding affected by these advances: completeness and spatial resolution. Focusing on 2005 birth and death certificate data from the State of North Carolina, we explore our ability to construct complete spatial datasets for large population-level administrative datasets at highly resolved spatial scales. If we are able to geocode administrative datasets thoroughly and at refined spatial scales, this will have important implications for the spatial scale at which public health research can be undertaken. Using data for the entire state, we assess geocoding completeness, or match rates, at different spatial resolutions across various subpopulations and regions.

\section{Methods}

\subsection{Data}

We used two large administrative datasets from North Carolina (NC) - the detail birth record (DBR) and the detail death record (DDR). Through a data sharing agreement, the NC State Center for Health Statistics provided individually-identified birth and death record data with key demographic, health, and geographic variables for all births and deaths occurring in North Carolina in 2005. Each record provided geographic information that was collected at the time of the birth or death, including county of residence, ZIP code, and street address. Note that 


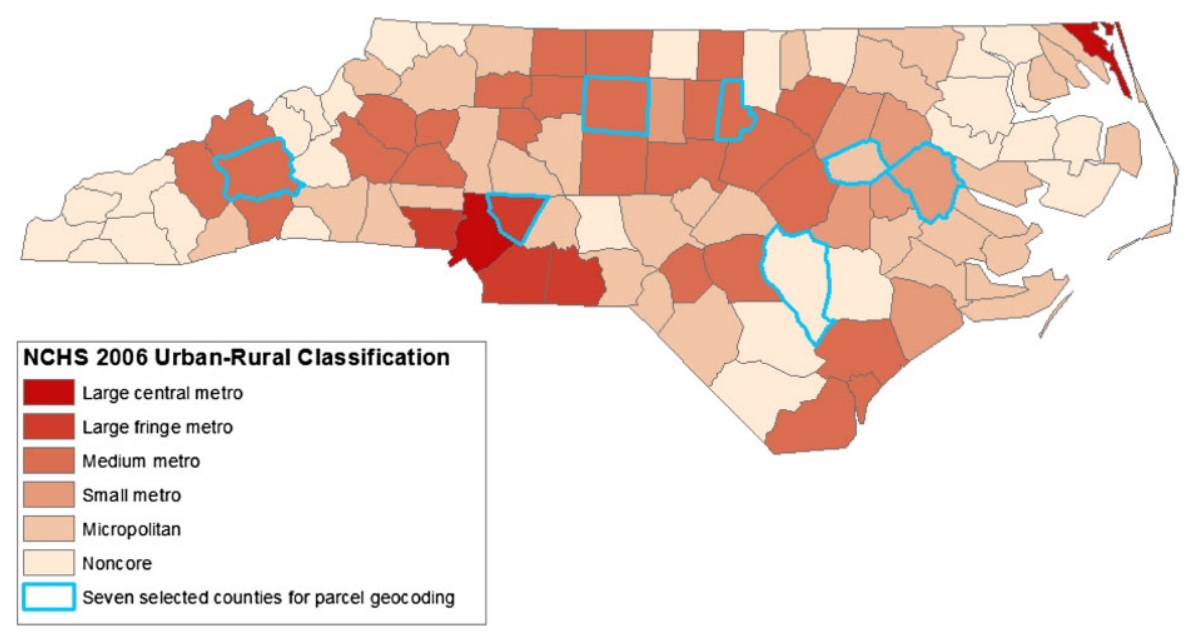

Figure 1 County urban-rural designations as classified by the National Center for Health Statistics (NCHS) and the seven counties selected for parcel geocoding

the street address provided in the DBR is actually the mailing address, with a variable to indicate if this was also the residential address. Quality of the DBR data has been studied and reported elsewhere (Buescher et al. 1993; Vinikoor et al. 2010), with studies indicating demographic and outcome data is fairly well recorded. No studies were available on the quality of the DDR data.

Race, age, education, marital status, and select health outcome variables provided in the DBR and DDR allowed us to examine trends in geocoding rates in the state overall, as well as within demographic subgroups. Births and deaths were subsetted by race into non-Hispanic white (NHW), non-Hispanic black (NHB), Hispanic (H), and Asian/Pacific Islander (A/PI); all records falling into other race categories were dropped from race-stratified analysis due to small sample size. Educational attainment of the decedent (DDR) and of the mother (DBR) was classified as less than $9^{\text {th }}$ grade, some high school, high school degree, some college, and college degree+. For the DBR, maternal age was classified into 5-year age groups for those women aged 15-44. For the DDR, the age of the decedents was classified as <20 years, 20-29 years, 30-39 years, $40-49$ years, $50-59$ years, $60-69$ years, $70-79$ years, $80-89$ years, and $\geq 90$ years. Preterm birth ( $<37$ weeks gestational age at delivery) and diabetes-related death were selected as example public health outcomes to which geospatial analysis might be applied.

In addition, we used data from the National Center for Health Statistics (NCHS) to assign each birth and death an urbanization level based on the reported county of residence. The NCHS classifies all US counties into one of six levels of urbanization: large central metro, large fringe metro, medium metro, small metro, micropolitan (nonmetro), and noncore (nonmetro) (US Centers for Disease Control and Prevention et al. 2012). Figure 1 shows the distribution of these urbanization levels across NC.

\subsection{Geocoding}

Residential addresses were geocoded using Esri ArcGIS 9.3 (Redlands, California) at three levels of spatial resolution - ZIP code, street, and parcel. Figure 2 displays how the same address would be spatially located using the three methods. Geocoding to an areal unit (e.g. 


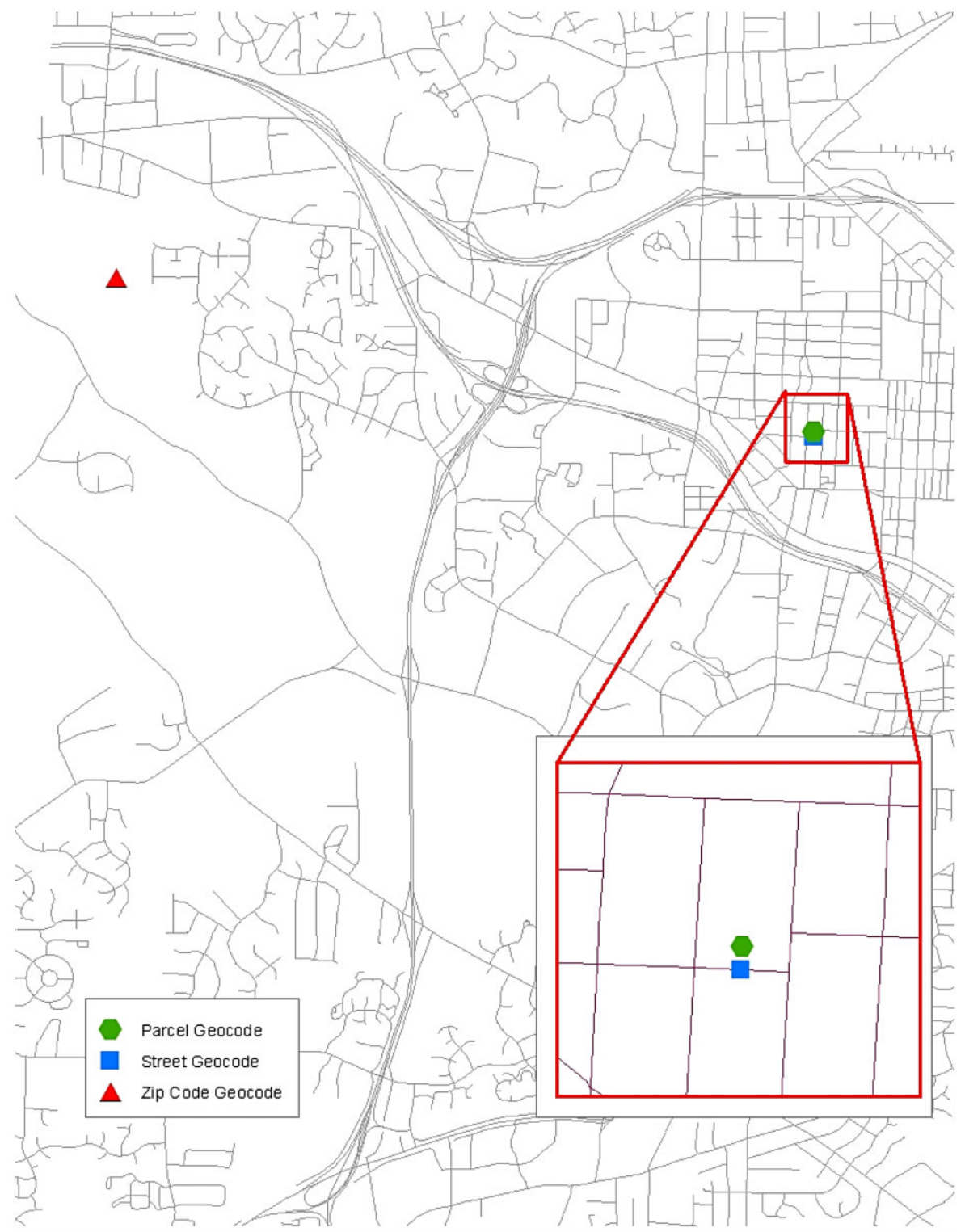

Figure 2 Differences in assigned location of an address using three different geocoding methods

ZIP code or parcel) typically assigns an address the latitude and longitude of the centroid of the areal unit with the corresponding address information. Street geocoding places the observation proportionally along a street segment, taking into account the address range of the street segment and the street number of the address. Rushton et al. (2006) provide a more in-depth description of how spatial location is assigned during the geocoding process.

The US Census Zip Code Tabulation Area 2000 geography (US Census Bureau 2012) was used as the reference layer for ZIP code level geocoding. Only records with a ZIP code that exactly matched a ZIP code in the reference layer were geocoded. 
Street level geocoding located residential street addresses based on the 2006 Topologically Integrated Geographic Encoding and Referencing system (TIGER) street centerlines file (US Census Bureau and Geography Division 2007). Similar to the manual geocoding improvement approach (Goldberg et al. 2008), street level geocoding occurred in three batches. First, we geocoded addresses that exactly matched to the road reference layer's street address components. Unmatched addresses from this first batch were standardized to be more compatible with the road reference layer by removing apartment, unit, suite, and lot numbers; formatting highways and routes numbers; and obtaining street addresses for common apartment complexes, housing developments, and mobile home parks. For the second batch, we geocoded those standardized addresses matching exactly to street address components in the reference layer. The final batch required a GIS technician to examine candidate matches for all remaining unmatched records and select candidate matches for geocoding when appropriate. An inexact match might be selected as the geocoded location for a DBR or DDR record due to, for example, spelling errors in an address; incorrect street type or direction; and units within housing developments, apartment complexes, or mobile home parks.

The parcel geocoding process referenced data provided by county tax assessor offices in order to assign records to individual tax parcels based on the physical address of each parcel (i.e. not the owner's mailing address). Geocoding to the tax parcel level was done using the same three tiered batch process described above (exact matches with original address, exact matches with standardized address, and hand matching).

ZIP code and street level geocoding were performed statewide for both the DBR and DDR data; however, parcel geocoding could not be undertaken statewide as not all NC counties have developed GIS layers of their tax parcel data and reporting of physical address within parcel data varies by county. The seven most rural counties in NC did not have any parcel GIS data, and rural counties also included physical address in parcel data less frequently than more urban counties. Thus, parcel geocoding of births and deaths was undertaken in seven select North Carolina counties: Buncombe, Cabarrus, Durham, Guilford, Pitt, Sampson, and Wilson. These counties are highlighted in Figure 1. While additional counties have developed tax parcel GIS layers, these seven counties were chosen for their distribution across the state and as examples of geographic areas with different levels of urbanization. In order from most urban to most rural, these counties are classified as large fringe metro (Cabarrus), medium metro (Buncombe, Durham, Guilford), small metro (Pitt), micropolitan (Wilson), and noncore (Sampson).

While ZIP code geocoding was undertaken for all records, street and parcel level geocoding were only undertaken for those records for which a residential street address was reported. Records were considered to be missing residential street address if the address field was blank, only a mailing address was provided, a partial street name was provided, a post office box was reported, or rural route box was reported. Missing addresses were identified manually. The inclusion of a residential street address in a record does not guarantee the address can be geocoded. Records may not be able to be geocoded due to incomplete residential address (e.g. missing street direction or type), incomplete reference layer data, or inability to determine the address of a multi-residence facility. The geocoding match rate is defined as the proportion of all records (both those reporting and those not reporting an address) that were able to be successfully assigned a spatial location at a specific spatial resolution.

\subsection{Statistical Analysis}

We tested for differences in reporting of residential street address and geocoding match rates across demographic (race, age, education, marital status, and urbanization) and outcome 
(preterm birth for births and diabetes-related death for deaths) subgroups using Chi-squared tests. For both births and deaths, differences in address reporting and geocoding rates were assessed for each level of geocoding - ZIP code, street, and parcel (select counties only). Since this analytic approach required conducting a large number of tests, we would expect to find significant results purely by chance. To address this issue and be more confident that our results highlight true differences in reporting and geocoding rates, we calculated adjusted p-values using the false discovery rate (FDR) method of controlling error under multiple comparisons. This method controls for the expected proportion of tests that are incorrectly identified as significant, and attempts to balance the desire for power to identify true difference and the desire to be conservative in falsely identifying tests as significant. For the DBR and DDR analyses separately, the false discovery rate was controlled at 0.05 , thus tests with adjusted p-values less than 0.05 were considered significant and we could expect that, on average, $5 \%$ of the differences found may not actually be significant.

\section{Results}

In 2005, there were 121,248 births and 73,016 deaths reported in North Carolina's detailed birth and death records, respectively. Tables 1 and 2 present the distribution of births and deaths, respectively, across demographic subgroups, as well as residential street address reporting rates within each group. Most births were to mothers who were NHW, less than 30 years old, married, and with at least a high school education. Most deaths were of individuals who were 60 years of age or older, not married, and with at least a high school education. Both births and deaths were most commonly among residents of medium metro and micropolitan counties.

\subsection{Address Reporting}

The proportion of records that included a residential street address represent an upper limit on geocoding match rates for street and parcel level geocoding efforts (ZIP code geocoding only requires a ZIP code, not a full street address). Statewide, $92.7 \%$ of birth records and $99.2 \%$ of death records included a residential street address. In the DBR, missing addresses were predominantly due to reporting of mailing address rather than residential address (95\%). In the death data, post office and rural route boxes accounted for almost half of the records missing addresses, with partial addresses accounting for $42 \%$ and no street address information for $8 \%$ of these records.

Birth and death records from more rural areas were less likely to include a street address (FDR-adjusted $\mathrm{p}<0.05$ ); however, while address reporting rates in the death record remained above $98 \%$ in all urban-rural classifications, the reporting rate in the birth record dropped to as low as $73.5 \%$ in non-core counties. Address reporting among births varied by maternal race, age, education, and marital status (FDR-adjusted $\mathrm{p}<0.5$ ). Generally, births to women at higher risk for adverse pregnancy outcomes - minority women, extremely young or old mothers, women with low educational attainment, and single women - were less likely to report a street address in the DBR. Preterm births were also slightly less likely to report a street address than were term births $(92.9 \%$ for term versus $92.0 \%$ for preterm births; FDRadjusted $\mathrm{p}<0.05)$. In the death record data, although there were differences in residential street address reporting by race, age, marital status, and education (FDR-adjusted $\mathrm{p}<0.5$ ), differences across subgroups were small. 


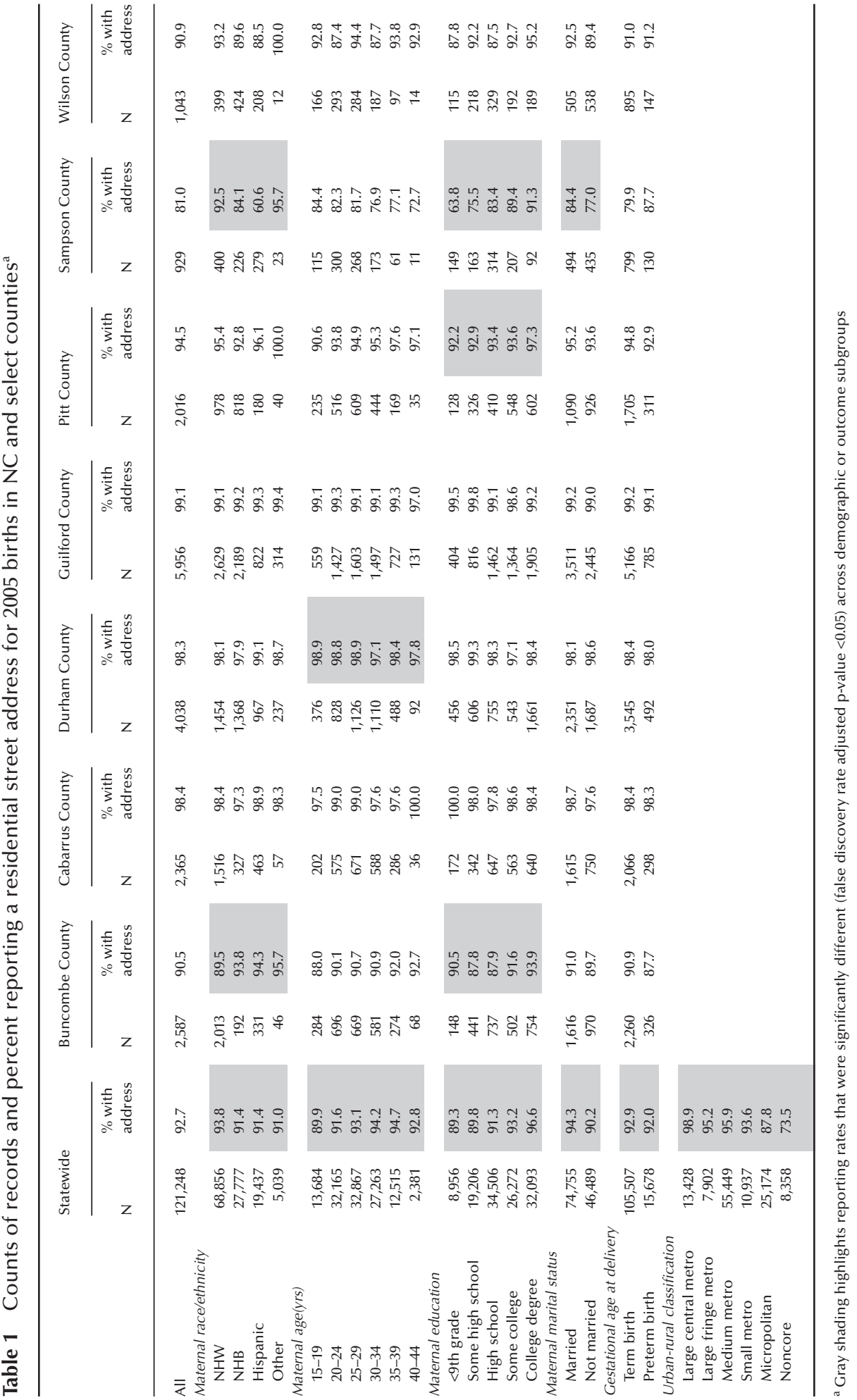




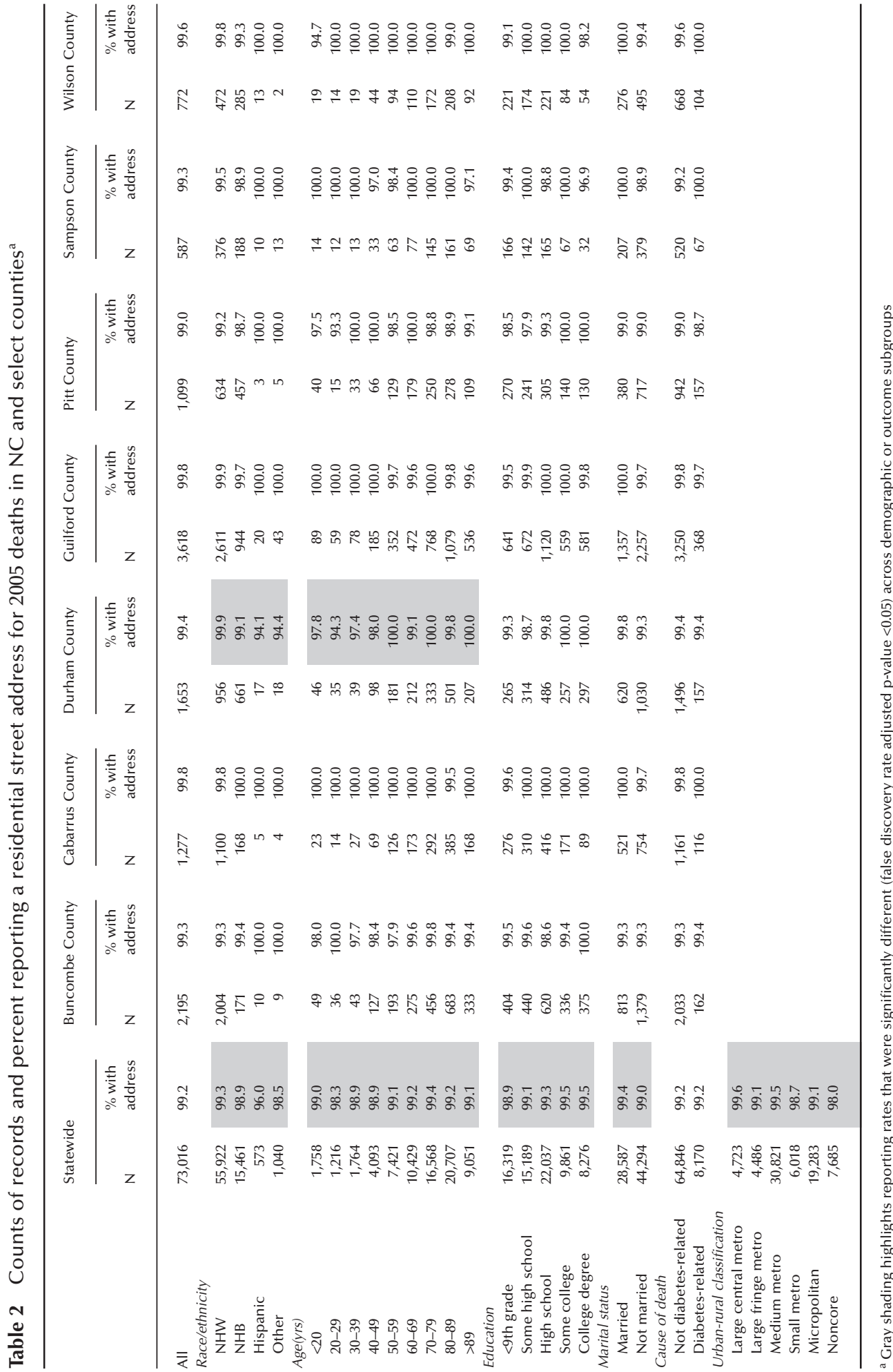


Among the seven counties selected for parcel geocoding, residential street address reporting in the birth data generally followed the statewide pattern of more urban areas having higher reporting rates than more rural areas; however, address reporting among births in Buncombe County, a medium metro area, was more on par with Wilson County, a micropolitan area, than with other medium metro counties. Among births in Sampson and Buncombe Counties, differences in address reporting across race/ethnicity were observed, with Hispanic women in Sampson County and NHW women in Buncombe County having lower levels of address reporting (FDR-adjusted $\mathrm{p}<0.5$ ). In Durham County, births to older mothers were less likely to report a street address (FDR-adjusted $\mathrm{p}<0.5$ ). In Buncombe, Pitt, and Sampson Counties, reporting rates in the DBR generally increased with education (FDR-adjusted $\mathrm{p}<$ $0.5)$. In all seven of these counties, address reporting rates for the death record were at least 99\%. Reporting rates among younger decedents and those of Hispanic ethnicity were lower in Durham County (FDR-adjusted $\mathrm{p}<0.5$ ), but no other differences in address reporting across demographic and outcome subgroups within these counties were noted.

\subsection{ZIP Code Geocoding}

ZIP code geocoding rates were high (see Tables 3 and 4), with $96.8 \%$ of births and $97.7 \%$ of deaths able to be geocoded at this level of spatial resolution. While geocoding rates did vary by race, age, education (in DDR only), marital status, and urbanization (FDR-adjusted $\mathrm{p}<0.5$ ), differences across subgroups were small, within \pm 3 percentage points. Among the seven counties selected for closer examination, Buncombe County had the lowest ZIP code geocoding rate for births at $96.5 \%$ and Durham County had the lowest rate for deaths at $97.5 \%$. In Durham County, small differences in ZIP code geocoding rates across race, age, and education were observed (FDR-adjusted $\mathrm{p}<0.5$ ).

Over 500 ZIP codes reported in either the DBR or DDR were not able to be geocoded. ZIP codes were not geocoded because they were not in NC $(42 \%$ of the unmatched ZIP codes), not real ZIP codes (16\% of the unmatched ZIP codes), or not found in the NC ZCTA data $(42 \%$ of the unmatched ZIP codes). Non-geocoded ZIP codes that actually exist in NC were distributed across the state, occurring in 67 of the $100 \mathrm{NC}$ counties; however, there were clusters of these ZIP codes in the most urban areas of NC, including Mecklenburg, Wake, Forsyth, and Guilford Counties. These ZIP codes may have been established since the 2000 ZCTA data were created.

Births and deaths in counties with parcel data that included data on the physical address of each parcel (i.e. could potentially be used for geocoding) were slightly less likely to be geocoded at the ZIP code level than births and deaths in counties lacking parcel data. For example, 96.3\% of births in counties with parcel data that included an address were geocoded, while $97.1 \%$ of births in counties without such data were geocoded. This may reflect the creation of new ZIP codes in urban areas that typically have more complete parcel data.

\subsection{Street Geocoding}

County street-level geocoding rates for both the DBR and DDR data are displayed in Figures $3 \mathrm{a}-\mathrm{b}$. The maps are drawn on the same legend using a monochromatic chloropleth in which higher geocoding rates are represented by darker shading and lower geocoding rates are represented by lighter shading. As highlighted by the darker overall color of the map in Figure $3 \mathrm{~b}$ (death data) compared to the map in Figure 3a (birth data), street geocoding rates were higher for the death record $(93.2 \%)$ than the birth record $(88.0 \%)$. Both maps also 


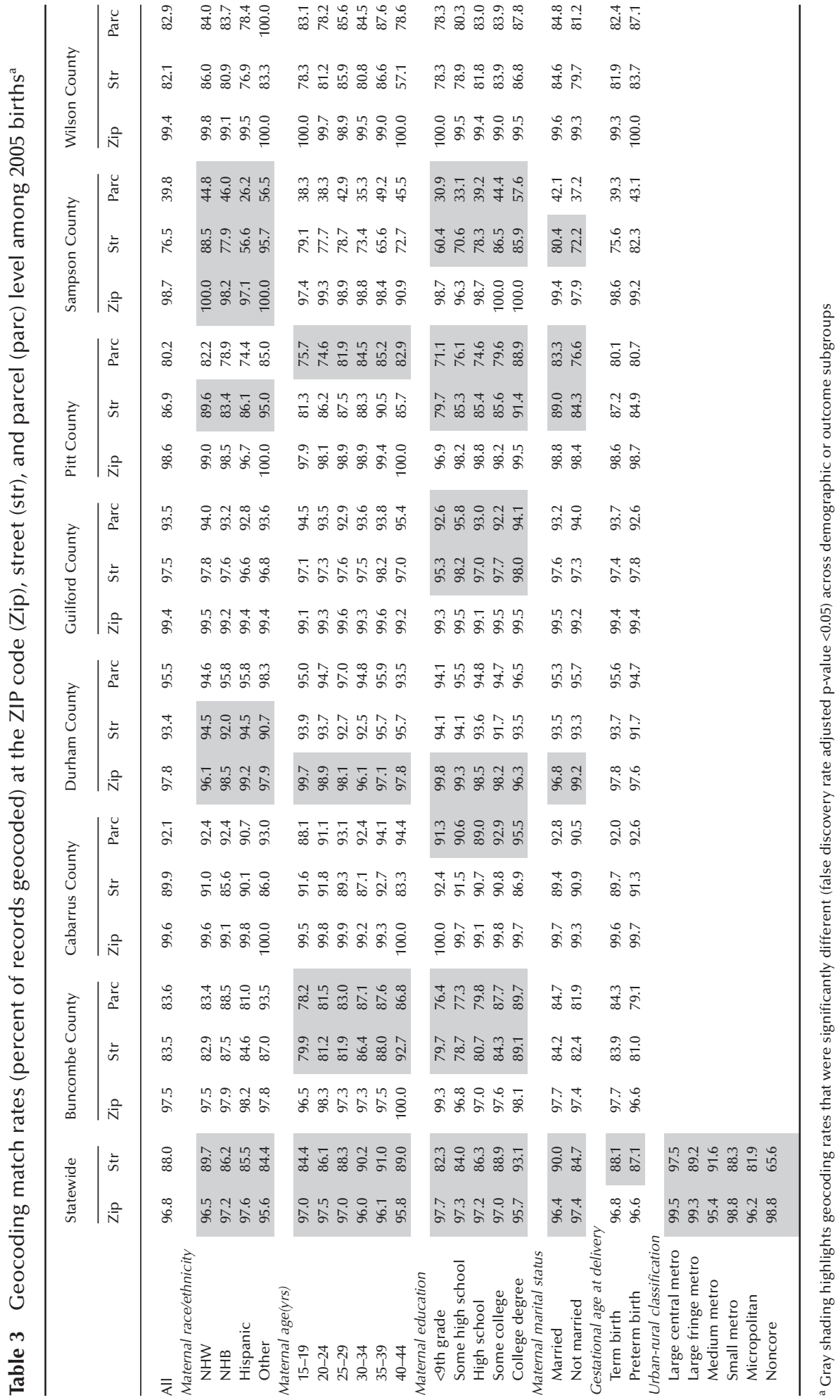




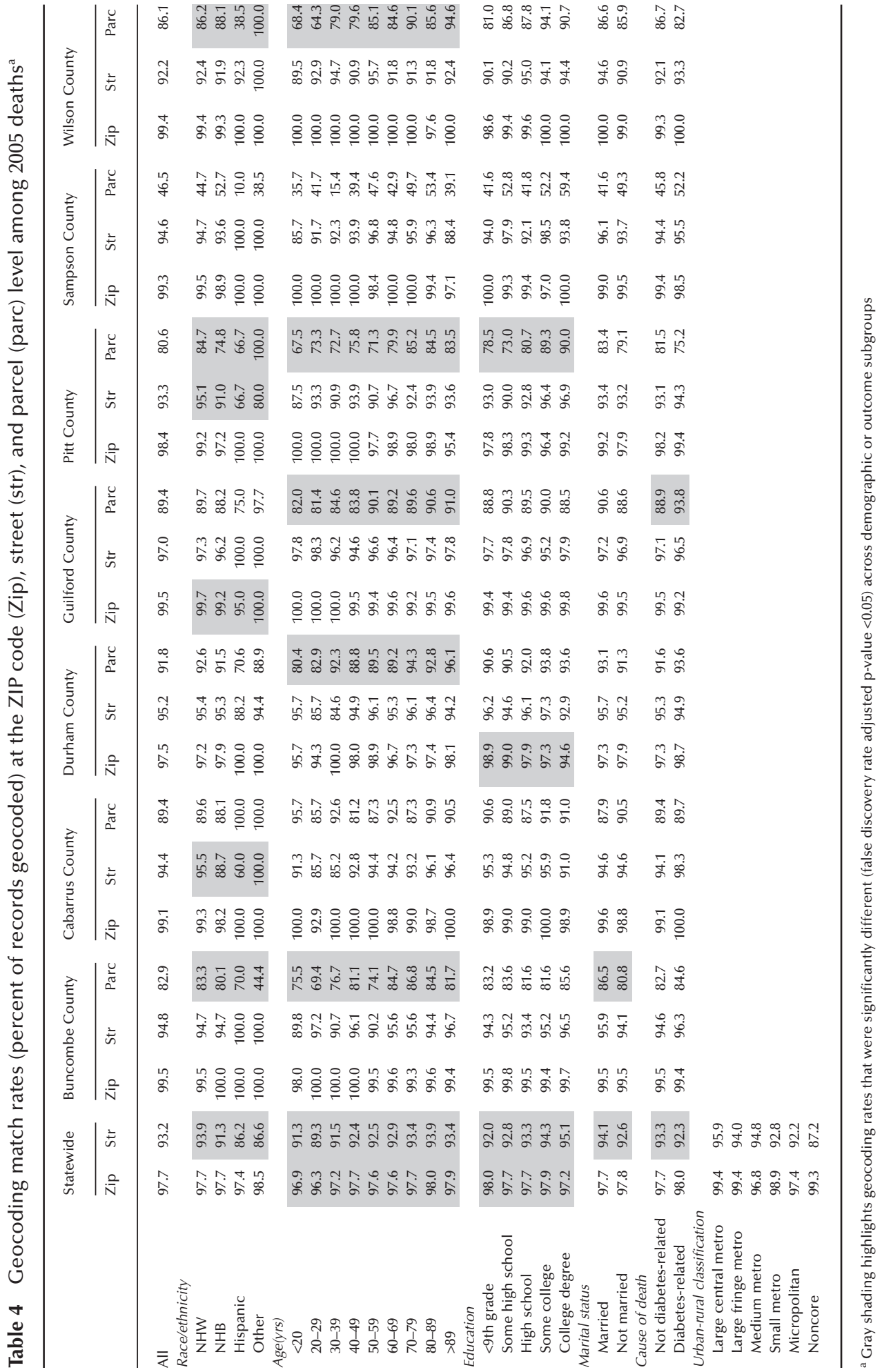



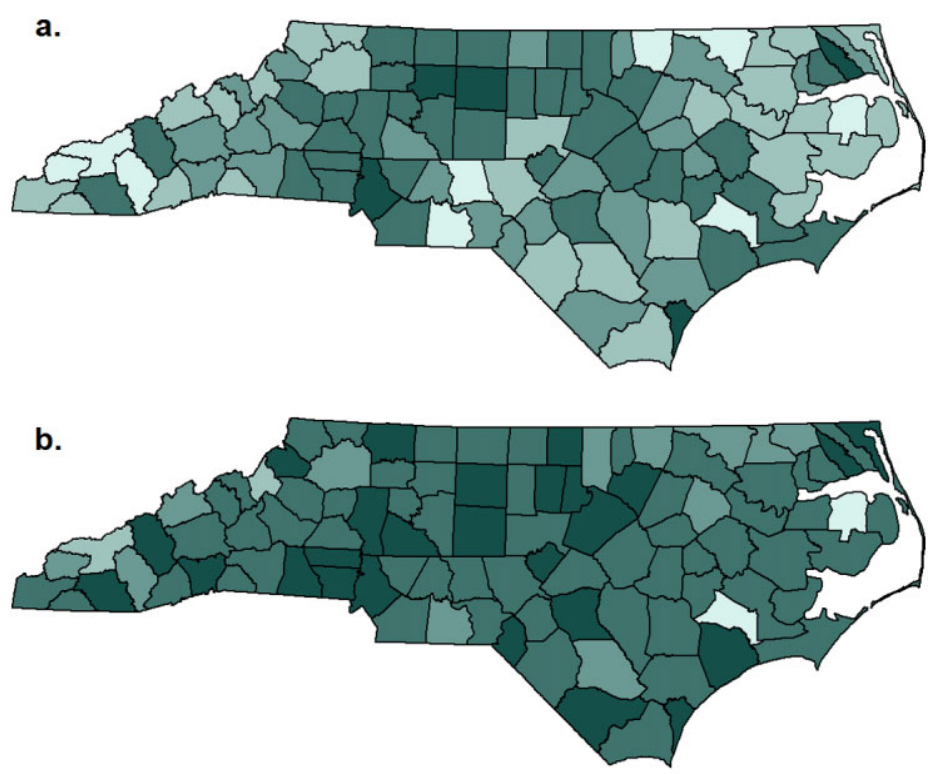

\begin{tabular}{c} 
County street geocoding rates (\% matched) \\
$<50$ \\
$50-75$ \\
\hline $50-85$
\end{tabular}

Figure 3 County street geocoding rates for 2005 NC births (a) and deaths (b)

reveal geographic variation in street-level geocoding rates across the state. This geographic variation is at least partially due to urbanization, which was significantly associated with geocoding rates for both datasets (FDR-adjusted $\mathrm{p}<0.5)$. Similar spatial patterning was not observed for county ZIP code level geocoding rates; county street and ZIP code level geocoding rates were not correlated.

Street geocoding rates for the entire state also varied by race, age, education, and marital status (FDR-adjusted $\mathrm{p}<0.5$ ). Generally, minority race, lower educational attainment, younger age, not being married, and residence in more rural areas were associated with lower geocoding rates. Street geocoding rates for the DBR data were also slightly higher for term $(88.1 \%)$ than for preterm $(87.1 \%)$ births (FDR-adjusted $\mathrm{p}<0.5)$, and rates for the DDR data were slightly higher for not diabetes-related $(93.3 \%)$ than for diabetes-related $(92.3 \%)$ deaths (FDR-adjusted $\mathrm{p}<0.5$ ). Across the state, street geocoding rates for both births and deaths were slightly lower in counties with parcel data that included data on the physical address of parcels than births and deaths in counties without such parcel data.

Although street geocoding rates for the seven selected counties generally followed the statewide pattern of higher geocoding rates in more urban areas and lower geocoding rates in more rural areas, Cabarrus County, the most urban of the seven counties, had only the third highest geocoding rate for births and fourth highest geocoding rate for deaths. Street geocoding rates for the birth data were quite disparate across the seven counties, ranging from $97.5 \%$ in Guilford County to $76.5 \%$ in Sampson County. The range of geocoding rates for the death data, on the other hand, was much smaller, ranging from $97.0 \%$ in Guilford County to $92.2 \%$ in Wilson County.

Within the counties, differences in geocoding rates were observed across each of the demographic variables considered. Geocoding rates for Hispanics were significantly lower than 
other race/ethnicity groups for deaths in Cabarrus and Pitt Counties, as well as births in Sampson County (FDR-adjusted $\mathrm{p}<0.05)$. On the other hand, geocoding rates were lowest for NHBs among births in Pitt and Durham Counties (FDR-adjusted $p<0.05$ ). Street geocoding rates increased with maternal age among Buncombe County births (FDR-adjusted $\mathrm{p}$ $<0.05)$. Lower educational attainment was associated with lower geocoding rates for births in Buncombe, Guilford, Pitt, and Sampson Counties (FDR-adjusted p $<0.05$ ). Births to married mothers were more likely to be street geocoded in Pitt and Sampson Counties (FDR-adjusted p $<0.05)$.

\subsection{Parcel Geocoding}

Since tax parcel GIS layers with address information are not available for the entire State of North Carolina, parcel geocoding was only undertaken in the seven selected counties. As was the case with street geocoding rates, parcel geocoding rates for each of the seven counties followed the general pattern of geocoding rates increasing with urbanization. In Sampson County, the most rural of the seven counties, parcel geocoding rates lagged far behind the other counties at $39.8 \%$ for births and $46.5 \%$ for deaths. Parcel geocoding rates in all other counties were at least $80 \%$ for both datasets.

Parcel geocoding was far less successful among Hispanics than among NHWs and NHBs for Sampson County births and Buncombe, Pitt, and Wilson County deaths (FDR-adjusted p < 0.05). Geocoding rates generally increased with age for deaths of residents of Buncombe, Durham, Guilford, Pitt, and Wilson Counties, and with maternal age for births to residents of Buncombe and Pitt Counties (FDR-adjusted $\mathrm{p}<0.05$ ). Geocoding rates varied by education among births in Buncombe, Cabarrus, Guilford, Pitt, and Sampson Counties, as well as among deaths in Pitt County (FDR-adjusted $\mathrm{p}<0.05$ ). Marital status was associated with geocoding rate for Buncombe County deaths and Pitt County births (FDR-adjusted $p<0.05$ ). Finally, in Guilford County, records for deaths related to diabetes were more likely to be parcel geocoded than records for deaths not related to diabetes $(93.8 \%$ vs. $88.9 \%$, respectively; FDR-adjusted $\mathrm{p}<0.05)$.

\section{Discussion}

Population-level administrative datasets contain vast amounts of important public health information. Converting these databases into spatial datasets through geocoding also allows administrative data to be linked with key environmental and social exposure data that could not be linked using traditional aspatial data architectures. However, public health researchers can only take advantage of the advances in GIS and spatial statistics if key datasets can be successfully geocoded at appropriately refined spatial scales.

In this article, we investigate geocoding completeness of public health data at the population scale and highlight issues related to geocoding completeness that may impact research relying on geocoded data. We find that population-level geocoding of administrative birth and death records for North Carolina can be undertaken at highly resolved spatial scales with high geocoding completeness. The minimum geocoding rate for producing a reliable spatial dataset has been established at $85 \%$ (Ratcliffe 2004), and our ZIP and street geocoding rates exceed or approach this mark in nearly all cases. Our geocoding rates at the ZIP code and street level were on par with previous studies (Krieger et al. 2002; McElroy et al. 2003; Zandbergen 2008), and our parcel rates, often over $80 \%$ and even $90 \%$, were quite high compared with 
previous work in which parcel geocoding rates rarely exceeded 70\% (Zandbergen 2008). Our parcel geocoding rates did, however, fall below the $85 \%$ mark in more rural counties where parcel reference layers are less fully developed.

These findings have implications for the spatial resolution at which population-level public health research is undertaken. Traditionally, public health research has focused on large geographic scales such as county, ZIP code, or Census tract (Rushton et al. 2006; Zandbergen and Chakraborty 2006) because geocoding to more highly resolved scales was difficult. We know, however, that the spatial resolution at which health research is conducted has important implications for our understanding of health and place (Moore and Carpenter 1999; Krieger et al. 2002; Dolinoy and Miranda 2004; Rushton et al. 2006; Leonard et al. 2011; Root 2012). Analysis at coarse spatial resolution may obscure within-unit spatial heterogeneity, hiding relationships that become apparent when analyses are conducted at a finer spatial resolution (Krieger et al. 2002; Dolinoy and Miranda 2004; Billaudeau et al. 2011; Leonard et al. 2011; Root 2012). In addition, exposures of interest may not coincide with the boundaries defined by governmental organizations and may vary at short distances (Rushton et al. 2006; Root 2012). The high geocoding match rates we achieved for population-level birth and death data indicate that it is possible to create high quality, highly resolved geocoded data layers from large administrative datasets. Such datasets can support research in which analytical approaches are determined based on the research question of interest rather than driven by limitations of the spatial data available. We thus support routinely geocoding to the highest spatial resolution feasible in order to provide researchers with the flexibility to aggregate data in a variety of ways to support the broadest range of analyses (Rushton et al. 2006; Leonard et al. 2011).

While we were able to geocode at highly resolved spatial scales, which can support spatial analysis at more refined geography, it is important to note that we did observe differences in geocoding success across subpopulations, which can affect the interpretation and generalizability of analytical findings based on the resulting datasets (Krieger et al. 2001; Oliver et al. 2005). Importantly, we noted differences in our ability to geocode data across health outcomes. In the birth data, address reporting was slightly lower for preterm births than for term births $(92.0 \%$ vs. $92.9 \%)$. Street geocoding rates were slightly higher for term births than preterm births $(88.1 \%$ vs. $87.1 \%)$, although there was considerable variability across counties (e.g. street geocoding match rates were higher for preterm births in four of the seven counties we investigated in detail). Generally, parcel geocoding rates were higher for term births than for preterm births, although not so in two of the seven detailed counties. Results varied similarly in geocoding match rates for non-diabetes-related deaths and diabetesrelated deaths. In the death data, statewide street geocoding rates were one percentage point higher for deaths not related to diabetes than for deaths related to diabetes.

In addition, geocoding rates were lower for disadvantaged and/or higher risk demographic subgroups. The largest differences we observed were in our ability to geocode at the street level across urbanization categories. For example, statewide, just $65.5 \%$ of births in noncore counties were able to be street geocoded, compared to $81.9 \%$ of births in the next most rural counties (micropolitan areas) and $95.7 \%$ of births in the most urban counties (large central metros). This difference in geocoding success by urbanization, which has also been noted in previous studies (Bonner et al. 2003; Cayo and Talbot 2003; McElroy et al. 2003), reflects the more complete reference layers in more urban areas and indicates a need to help lowerresourced rural counties improve their GIS infrastructure. For other demographics, the magnitude of the differences in street geocoding rates across demographic subgroups was less than 10 percentage points for statewide data; although within some of the seven counties consid- 
ered in more detail, we did see some large differences in geocoding across race groups at the street and parcel levels, with births and deaths to Hispanics proving especially difficult to geocode, particularly in rural areas.

Research using geocoded data must be careful to note how variations in geocoding rates across key demographic groups and health outcomes may impact analytical findings and how these differences in geocoding rates may be pertinent to the outcomes under investigation (e.g. impact of residential instability, access to care, etc.)

We also note that geocoding rates were lower for the birth record than the death record data (e.g. street geocoding match rates of $88.0 \%$ and $93.2 \%$, respectively). This difference, however, does not reflect a true difference between the two datasets in our ability to locate addresses. Rather, the difference in the geocoding rates is primarily due to the higher rate of residential street address reporting in the death record. For example, if we only consider records that included a residential street address, then the proportion of NC birth and death records that could be street geocoded are actually quite similar at $94.9 \%$ and $93.9 \%$, respectively. Since we are interested in our ability to geocode these datasets at the population-level, we cannot ignore records with missing address information. This suggests the importance of improving reporting of residential street addresses in administrative datasets.

This study is not without limitations. For small subpopulations, such as very young decedents, certain minority groups, or very rural communities, small changes in the number of records may translate to large changes in address reporting rates and/or geocoding rates. Additionally, we only studied birth and death certificate data in North Carolina. The quality and consistency of reporting of address information in corresponding datasets may vary by state, and thus have important implications for geocoding these data. Other administrative datasets, even those covering North Carolina, such as immunizations, blood lead screening, disease registries, and educational outcomes may also vary in the ability to be successfully geocoded. Finally, we assess geocoding completeness at various spatial scales, but are not able to comment on the positional accuracy of geocoded locations. Positional accuracy is another important metric of geocoding quality, and may vary across the urban-rural spectrum, thus potentially compounding the differences in completeness we note across this same spectrum.

An additional caveat of this work is that we did not utilize some recent advances in geocoding technology; however, we believe many health researchers may have to make a similar choice. Advances in geocoding in the ArcGIS platform, including both web- and desktop-based services, may produce high quality geocoded datasets more quickly than the geocoding methodology we used here, but this may be at the expense of confidentiality and spatial resolution. While web-based geocoding services can be both fast and accurate, these technologies require that data be passed over a web connection to a remote server. Data sharing agreements and security protocols may not permit such sharing of confidential health data over the Internet. Data confidentiality concerns lead us to forego the web-based geocoding options in favor of traditional, locally-based geocoding methods. We acknowledge, however, that in instances when data confidentiality is not a concern, the recently developed web-based geocoding options and other geocoding products may produce quality geocoding results. In addition, advances have also been made in the desktop-based address locators included with the ArcGIS platform; however, these locators may geocode at lower levels of resolution, for example ZIP code or city level, if they cannot match a street address. Thus, use of these locators requires careful attention to their underlying methodology and to the interpretation of the resulting geocoded datasets. For this analysis, we selected our geocoding methodology, including the use of TIGER streets as our address reference layer, based on our desire 
to protect confidentiality and control spatial resolution. Furthermore, TIGER streets align spatially with Census boundaries, making it easier to spatially join our geocoded births and deaths with demographic and socioeconomic data for future research.

While we demonstrate high geocoding rates for both births and deaths at the population level and within key demographic subgroups, we were not able to geocode all records in either administrative dataset. In most datasets, there will inevitably be records that cannot be geocoded to the individual address level. Methods are being developed to combine data geocoded at different levels in order to allow researchers to use all records (Hibbert et al. 2009; Goovaerts 2012). Simultaneously pursing the further development of such methodologies and efforts to improve our ability to geocode administrative data at highly resolved spatial scales through improved reference layers and address reporting will create a multitude of new research opportunities in spatial public health analysis.

\section{Conclusions}

Overall, we find that we are able to successfully geocode population-level birth and death certificate data in North Carolina at highly resolved spatial scales. The spatial scales at which we were able to achieve high geocoding completeness allow for analysis at a more refined spatial scale than has traditionally been used for public health research. More spatially refined analyses allows for better characterization of associations between exposures and outcomes exhibiting spatial heterogeneity within larger areal units such as ZIP codes.

This work affirms that highly resolved spatial data architectures for population-level administrative public health datasets are viable through geocoding of street address information. We concur with previous work that has recommended geocoding to the highest spatial resolution feasible given consideration of the other dimensions of geocoding quality (completeness, accuracy, and matching algorithm criteria) (Rushton et al. 2006; Leonard et al. 2011). We recommend routinely geocoding administrative health data to the street level and, even more ideally, the parcel level where good quality reference data are available. As more administrative datasets are geocoded with high levels of completeness at highly resolved scales, the decision of the spatial resolution at which research is conducted need not be dictated by limitations of geocoding, but can instead be determined by our understanding of the biological processes and social and environmental exposures being investigated. Such research, however, must acknowledge how disparate geocoding success across subpopulations may affect findings.

\section{References}

Bell S, Wilson K, Shah T I, Gersher S, and Elliott T 2012 Investigating impacts of positional error on potential health care accessibility. Spatial and Spatiotemporal Epidemiology 3: 17-29

Bergquist R and Rinaldi L 2010 Health research based on geospatial tools: A timely approach in a changing environment. Journal of Helminthology 84: 1-11

Billaudeau N, Oppert JM, Simon C, Charreire H, Casey R, Salze P, Badariotti D, Banos A, Weber C, and Chaix B 2011 Investigating disparities in spatial accessibility to and characteristics of sport facilities: Direction, strength, and spatial scale of associations with area income. Health and Place 17: 114-21

Bonner M R, Han D, Nie J, Rogerson P, Vena J E, and Freudenheim J L 2003 Positional accuracy of geocoded addresses in epidemiologic research. Epidemiology 14: 408-12

Bow C J, Waters N M, Faris P D, Seidel J E, Galbraith P D, Knudtson M L, Ghali W A, and the APPROACH Investigators 2004 Accuracy of city postal code coordinates as a proxy for location of residence. International Journal of Health Geographics 3: 5 
Buescher P A, Taylor K P, Davis M H, and Bowling J M 1993 The quality of the new birth certificate data: A validation study in North Carolina. American Journal of Public Health 83: 1163-65

Cayo M R and Talbot T O 2003 Positional error in automated geocoding of residential addresses. International Journal of Health Geographics 2: 10

Comer K F, Grannis S, Dixon B E, Bodenhamer D J, and Wiehe S E 2011 Incorporating geospatial capacity within clinical data systems to address social determinants of health. Public Health Reports 126(Suppl. 3): 54-61

Dolinoy D C and Miranda M L 2004 GIS modeling of air toxics releases from TRI-reporting and nonTRI-reporting facilities: Impacts for environmental justice. Environmental Health Perspectives 112: 1717-24

Duncan D T, Castro M C, Blossom J C, Bennett G G, and Gortmaker S L 2011 Evaluation of the positional difference between two common geocoding methods. Geospatial Health 5: 265-73

Eisen L and Eisen R J 2011 Using geographic information systems and decision support systems for the prediction, prevention, and control of vector-borne diseases. Annual Reviews in Entomology 56: 41-61

Goldberg D W and Cockburn M G 2012 The effect of administrative boundaries and geocoding error on cancer rates in California. Spatial and Spatiotemporal Epidemiology 3: 39-54

Goldberg D W and Jacquez G M 2012 Advances in geocoding for the health sciences. Spatial and Spatiotemporal Epidemiology 3: 1-5

Goldberg D W, Knoblock C A, Wilson J P 2007 From text to geographic coordinates: The current state of geocoding. URISA Journal 19: 33-46

Goldberg D W, Wilson J P, Knoblock C A, Ritz B, and Cockburn M G 2008 An effective and efficient approach for manually improving geocoded data. International Journal of Health Geographics 7: 60

Goovaerts P 2012 Geostatistical analysis of health data with different levels of spatial aggregation. Spatial and Spatiotemporal Epidemiology 3: 83-92

Healy M A and Gilliland J A 2012 Quantifying the magnitude of environmental exposure misclassification when using imprecise address proxies in public health research. Spatial and Spatiotemporal Epidemiology 3: $55-67$

Hibbert J D, Liese A D, Lawson A, Porter D E, Puett R C, Standiford D, Liu L, and Dabelea D 2009 Evaluating geographic imputation approaches for ZIP code level data: An application to a study of pediatric diabetes. International Journal of Health Geographics 8: 54

Jacquez G M 2012 A research agenda: Does geocoding positional error matter in health GIS studies? Spatial and Spatiotemporal Epidemiology 3: 7-16

Krieger N, Chen J T, Waterman P D, Rehkopf D H, and Subramanian S V 2005 Painting a truer picture of US socioeconomic and racial/ethnic health inequalities: The Public Health Disparities Geocoding Project. American Journal of Public Health 95: 312-23

Krieger N, Chen J T, Waterman P D, Soobader M J, Subramanian S V, and Carson R 2002 Geocoding and monitoring of US socioeconomic inequalities in mortality and cancer incidence: Does the choice of areabased measure and geographic level matter? American Journal of Epidemiology 156: 471-82

Krieger N, Waterman P, Lemieux K, Zierler S, and Hogan J W 2001 On the wrong side of the tracts? Evaluating the accuracy of geocoding in public health research. American Journal of Public Health 91: 1114-16

Leonard T C, Caughy M O, Mays J K, and Murdoch J C 2011 Systematic neighborhood observations at high spatial resolution: Methodology and assessment of potential benefits. PLoS One 6: e20225

McElroy J A, Remington P L, Trentham-Dietz A, Robert S A, and Newcomb P A 2003 Geocoding addresses from a large population-based study: Lessons learned. Epidemiology 14: 399-407

McLafferty S, Freeman V L, Barrett R E, Luo L, and Shockley A 2012 Spatial error in geocoding physician location data from the AMA Physician Masterfile: Implications for spatial accessibility analysis. Spatial and Spatiotemporal Epidemiology 3: 31-8

Mindell J and Barrowcliffe R 2005 Linking environmental effects to health impacts: A computer modelling approach for air pollution. Journal of Epidemiology and Community Health 59: 1092-98

Miranda M L and Edwards S E 2011 Use of spatial analysis to support environmental health research and practice. North Carolina Medical Journal 72: 132-35

Moore D A and Carpenter T E 1999 Spatial analytical methods and geographic information systems: Use in health research and epidemiology. Epidemiologic Reviews 21: 143-61

Oliver M N, Matthews K A, Siadaty M, Hauck F R, and Pickle L W 2005 Geographic bias related to geocoding in epidemiologic studies. International Journal of Health Geographics 4: 29

Ratcliffe J H 2001 On the accuracy of TIGER-type geocoded address data in relation to cadastral and census areal units. International Journal of Geographical Information Science 15: 473-85

Ratcliffe J H 2004 Geocoding crime and a first estimate of a minimum acceptable hit rate. International Journal of Geographical Information Science 18: 61-72 
Robinson J C, Wyatt S B, Hickson D, Gwinn D, Faruque F, Sims M, Sarpong D, and Taylor H A 2010 Methods for retrospective geocoding in population studies: The Jackson Heart Study. Journal of Urban Health 87: 136-50

Root E D 2012 Moving neighborhoods and health research forward: Using geographic methods to examine the role of spatial scale in neighborhood effects on health. Annals of the Association of American Geographers 102: 986-95

Rushton G, Armstrong M P, Gittler J, Greene B R, Pavlik C E, West M M, and Zimmerman D 2006 Geocoding in cancer research: A review. American Journal of Preventive Medicine 30: S16-S24

US Census Bureau 2012 Census 2000 5-Digit ZIP Code Tabulation Areas (ZCTAs) Cartographic Boundary Files. Washington, DC, US Census Bureau

US Census Bureau, Geography Division 20072006 Second Edition TIGER/Line Files. Washington, DC, US Census Bureau

US Centers for Disease Control and Prevention, National Center for Health Statistics, Office of Analysis and Epidemiology 2012 NCHS Urban-Rural Classification Scheme for Counties 2006. Atlanta, GA, US Centers for Disease Control and Prevention

Vinikoor L C, Messer L C, Laraia B A, and Kaufman J S 2010 Reliability of variables on the North Carolina birth certificate: A comparison with directly queried values from a cohort study. Paediatric and Perinatal Epidemiology 24: 102-12

Ward M H, Nuckols J R, Giglierano J, Bonner M R, Wolter C, Airola M, Mix W, Colt J S, and Hartge P 2005 Positional accuracy of two methods of geocoding. Epidemiology 16: 542-47

Zandbergen P A 2008 A comparison of address point, parcel and street geocoding techniques. Computers, Environment and Urban Systems 32: 214-32

Zandbergen P A and Chakraborty J 2006 Improving environmental exposure analysis using cumulative distribution functions and individual geocoding. International Journal of Health Geographics 5: 23 